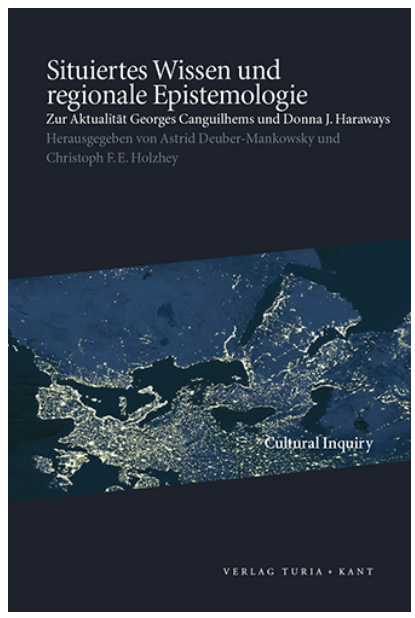

Situiertes Wissen und regionale Epistemologie: Zur Aktualität Georges Canguilhems und Donna J. Haraways, ed. by Astrid Deuber-Mankowsky and Christoph F. E. Holzhey, Cultural Inquiry, 7 (Wien: Turia + Kant, 2013), pp. 35-49

\section{JEAN-FRANÇOIS BRAUNSTEIN}

\section{Die Geschichte des Regionsbegriffs in der Epistemologie}

\author{
CITE AS:
}

Jean-François Braunstein, »Die Geschichte des Regionsbegriffs in der Epistemologie «, in Situiertes Wissen und regionale Epistemologie: Zur Aktualität Georges Canguilhems und Donna J. Haraways, hg. v. Astrid Deuber-Mankowsky und Christoph F. E. Holzhey, Cultural Inquiry, 7 (Wien: Turia + Kant, 2013), S. 35-49 <https://doi.org/10.37050/ ci-07_02>

RIGHTS STATEMENT:

(C) by the author(s)

This version is licensed under a Creative Commons AttributionShareAlike 4.0 International License. 


\title{
DIE GESCHICHTE DES REGIONSBEGRIFFS IN DER EPISTEMOLOGIE
}

\author{
Jean-François Braunstein
}

So sehr der Begriff des »situierten Wissens « zur Zeit in Mode ist, so sehr wirkt der Begriff der »Region « veraltet, ja sogar ein wenig überholt, ruft doch der »epistemologische Regionalismus « in Frankreich die 1960er und 1970er Jahre in Erinnerung: die Jahre Bachelards, Canguilhems und Foucaults. Doch die Tatsache, dass 2011 am ICI Berlin Institute for Cultural Inquiry eine Tagung zum Thema »Situiertes Wissen und regionale Epistemologie « stattfand, belegt ohne Zweifel, dass diese Fragestellung das Verfallsdatum doch noch nicht überschritten hat. Kaum mehr aufgegriffen wird dieses Thema in Frankreich selbst, wo man sich heute, mit einer gewissen Verspätung gegenüber der angelsächsischen Welt, eher mit der Frage der Einheit der Wissenschaften beschäftigt. So werden paradoxerweise die durch die »historische Epistemologie « aufgeworfenen Fragen stärker außerhalb als innerhalb jenes Landes diskutiert, das die Entstehung dieser Wendung schon vor langer Zeit erlebte. ${ }^{1}$ Eine historische Klärung der damit einhergehenden Fragen wäre sicherlich von Nutzen, um besser nachvollziehen zu können, aus welchem historischen, aber auch »geographischen « Kontext die Problematik der »situierten Wissensformen « hervorgegangen ist. Beide Traditionen - die relativ alten »regionalen Epistemologien « einerseits und die jüngeren »situierten Wissensformen « andererseits - können in mancherlei Hinsicht auch zusammenlaufen, wie ein Artikel von François Dagognet, dem letzten großen Vertreter der französischen Tradition des »epistemologischen Regionalismus ", bezeugt, der vor einigen Jahren in Science in Context und damit in einer Zeitschrift erschienen ist, die emblematisch für einen dem situierten Wissen verpflichteten Zugang steht. ${ }^{2}$ Im Titel dieses Artikels - »A Regional Epistemology with Possibilities for Expansion « - klingt bereits eine Art Programm an.

Es ist wohl sinnvoll, zunächst die Vorgeschichte des Begriffs der Region in der Epistemologie in aller Kürze darzustellen, bevor mit Gaston Bachelard und Georges Canguilhem die beiden Autoren ins Gedächtnis gerufen werden, die ihn am prominentesten verkörperten. 
Danach soll auf die Werke von Michel Foucault und Louis Althusser eingegangen werden, die diese Bezugnahme auf den Regionsbegriff erneuerten und zugleich transformierten. Es bietet sich des Weiteren an, die Kontexte dieser Verwendungen hinzuzuziehen, denn schließlich ist doch sicher das Mindeste, was man mit dem Begriff der Region tun kann, ihn zu kontextualisieren. Dabei wird sich zeigen, dass dieser »französische « Zugang zum Begriff der Region und die angelsächsische Frage nach der »Uneinheit « (disunity) der Wissenschaften sich in gewisser Hinsicht berühren.

\section{DIE VORGESCHICHTE EINES BEGRIFFS: KARTEN, OZEANE UND REGIONEN}

Eine Erkundung der Vorgeschichte des Regionsbegriffs wird bei Francis Bacon anzusetzen haben. Bei ihm findet sich erstmals der Vergleich des Wissens mit einer Karte und eine klare Ausformulierung der Vorstellung, dass sich Wissen räumlich repräsentieren lässt. So erklärt er in $D e$ dignitate et augmentis scientarum (1623), "gleichsam einen kleinen Globus der intellektuellen Welt geschaffen « zu haben, der die verschiedenen Teile des menschlichen Wissens beschreibt. ${ }^{3}$ In Neu-Atlantis klassifiziert er ferner die verschiedenen Wissensbereiche nach vertikalen Welt»Regionen «, die im »Haus des Salomon" studiert werden, das den Wissenschaften gewidmet ist: »Geräumige, tiefe Höhlen « sind der Erforschung der »Tiefere[n] Region«, »hohe Türme« der Erforschung der »Höhere[n] Region " gewidmet. $^{4}$

Bacon zufolge gibt es eine Entsprechung zwischen dem Globus des Wissens und dem Globus der Welt und es ist bekannt, dass er den Ehrgeiz hatte, eine Art Christoph Columbus des Denkens zu sein. Häufig zitiert er das alttestamentarische Verheißungswort "plurimi pertransibunt, et multiplex erit scientia « - »viele werden vorübergehen und von vielerlei Art wird das Wissen sein « (Daniel 12,4) - und überträgt es auf seine Zeit: »als ob die Eröffnung und das gründliche Durchstreifen der Welt und die Vermehrung des Wissens für das gleiche Zeitalter bestimmt seien $[\ldots] \ll .5$ Eine vollständige Landkarte des menschlichen Wissens müsste es demnach auch erlauben, die noch zu erforschenden Territorien besser zu umreißen. Die Metapher der Landkarte wäre genauer als die des Baumes: Dieser schreibt eine Hierarchie, eine relative Abhängigkeit zwischen den Wissenschaften vor, während die Karte die Möglichkeit einer nicht-hierarchischen Verteilung des Wissens offen lässt. 
Auf eben dieses Bild der Karte bezieht sich auch d'Alembert in seinem »Discours préliminaire « zur Encyclopédie, wenn er bezüglich der Enzyklopädie in eindeutig baconschem Ton erklärt: »Man könnte an eine Art Weltkarte denken, auf der die wichtigsten Länder, ihre Lage und ihre Abhängigkeit voneinander sowie die Verbindungen zwischen ihnen in Luftlinie verzeichnet sind. $\aleph^{6}$ Und wie bei allen Weltkarten gibt es auch hier, je nachdem, welcher Platz dem Menschen zugewiesen wird, verschiedene mögliche Kartennetze.

Eine zweite Metapher, die zur Repräsentation des Wissens verwendet wird, ist der Ozean. Insgesamt weniger verbreitet, findet sie dennoch prominent bei Leibniz Verwendung. Er vergleicht »den ganzen Körper der Wissenschaften « nicht mit einem Land, sondern vielmehr »mit einem Ozean [...], der überall stetig und ohne Unterbrechung oder Einteilung ist, wenn auch die Menschen in ihm Teile erdenken und ihnen Namen geben nach ihrer Bequemlichkeit «. Leibniz führt aus: »Und wie es unbekannte Meere gibt, auf denen nur einzelne Schiffe gesegelt sind, die der Zufall dorthin verschlug, so kann man ebenso sagen, daß es Wissenschaften gibt, von denen man nur durch Zufall und ohne Absicht etwas erfahren hat. " ${ }^{7}$ Mehr noch als die kartographische erlaubt es diese ozeanische Metapher die Kontinuität des Wissens zu betonen.

Die historisch betrachtet dritte Metapher zur räumlichen Repräsentation des Wissens macht schließlich von den Begriffen Region und Regionalismus Gebrauch. Im französischen Kontext taucht der Begriff des Regionalismus zunächst im Bereich des Politischen und zwar unter reaktionären Autoren auf. Oft wird der große nationalistische Schriftsteller Maurice Barrès (1862-1923) als Erfinder des Regionalismus angesehen, doch scheint der Ausdruck tatsächlich 1874 aus der Feder eines weitaus weniger bekannten Mannes hervorgegangen zu sein: des provençalischen Dichters Léon de Berluc-Pérussis (1835-1902), der ein Bewunderer von Le Play und Mitbegründer der »Fédération régionaliste française « war. Charles Maurras, Mitbegründer der royalistischen Bewegung »Action française «, war ebenfalls ein Verfechter des Regionalismus. Dies brachte er insbesondere in seiner Streitschrift »L'idee de la décentralisation « zum Ausdruck. Schließlich muss an dieser Stelle auch Henri Pourrat (1887-1959) erwähnt werden, ein regionalistisch gesinnter Schriftsteller, dem das Vichy-Regime sehr hohe Wertschätzung entgegenbrachte. Denn von ihm und seinem politischen Begriff des Regionalismus distanzierte sich Canguilhem in einem wenig bekannten Text aus dem Jahr $1929 .{ }^{8}$ 
Paradoxerweise bildete sich der Begriff der Region erst nach dem Begriff des Regionalismus heraus, und zwar insbesondere im Kreis der »École française de géographie humaine«. Für die Geographen dieses Kreises waren Regionen territoriale Einheiten, die sich um Einflusszentren herum organisieren, die von Großstädten gebildet werden. So sollte die Geographie von nun an nicht mehr von Grenzen, sondern von Organisationsgrenzen bestimmt werden. Auf diese Weise beschrieb Vidal de la Blache Frankreich in seinem Tableau de la géographie de la France von 1903. Derselbe Vidal de la Blache schlug im Jahre 1910 eine Verwaltungsreform vor, die Frankreich nach Regionen organisieren sollte. ${ }^{9}$ Eine Reihe von Historikern, die von der Humangeographie beeinflusst waren, stellten weitere Forschungen in diese Richtung an: In der von der Revue de synthèse historique herausgegebenen Reihe »Les régions de la France " veröffentlichte Lucien Febvre 1911 ein Buch mit dem Titel La Franche-Comté und Marc Bloch 1913 L'Île-de-France. Der junge Canguilhem zeigte sich an dieser Humangeographie und vor allen Dingen an ihrem Begriff der »Lebensweise " (genre de vie) sehr interessiert. Von diesem Begriff inspiriert notierte er, dass es nicht die Natur sei, die diese oder jene Lebensweise hervorbringe, sondern dass es im Gegenteil die Lebensweisen des Menschen seien, die »die Natur, so wie wir sie sehen «, hervorbringen. ${ }^{10}$ Der Begriff der Region erscheint hier durchweg als aufgewertet, allerdings wurde zugleich auch die dem Regionalismus von Beginn an beigegebene negative Konnotation nicht aus den Augen verloren. ${ }^{11}$

\section{BACHELARD UND DIE DEBATTEN ÜBER DEN RATIONALISMUS}

Bachelard ist der erste, der den Begriff des »regionalen Rationalismus « in Le rationalisme appliqué von 1949 verwendete. Seit dem Erscheinen von Der neue wissenschaftliche Geist (Le nouvel esprit scientifique) im Jahr 1934 hatte er, inspiriert von den Fragen der zeitgenössischen Wissenschaft und gegen die Lehre einer »allgemeine[n] und unveränderliche[n] Vernunft « in der Art von Émile Meyerson, die Bedeutung eines »offenen Rationalismus « unterstrichen. ${ }^{12}$ Zugleich beabsichtigte er im Jahre 1936, einen »Suprarationalismus « zu begründen, der sich »polemisch " und "a posteriori « gegen einen »Schulrationalismus « wenden sollte: »Man muss der menschlichen Vernunft die Funktion der Verwirrung und Aggressivität zurückerstatten. «13 Dabei ist anzumerken, dass dieser Artikel in der surrealistischen Zeitschrift Inquisitions neben den 
Artikeln von Roger Caillois, Tristan Tzara und René Crevel erschien. Bachelard verwies im Übrigen in seinem Aufsatz direkt auf Tristan Tzara: »Man wird eine experimentelle Vernunft begründen, die in der Lage ist, die Wirklichkeit ebenso suprarational zu organisieren, wie der experimentelle Traum Tristan Tzaras die dichterische Freiheit surrealistisch organisiert. « ${ }^{14} 1940$ beschließt er Die Philosophie des Nein (La philosophie du non) mit dem Satz: »Die traditionelle Lehre von der absoluten und unveränderlichen Vernunft stellt nur eine Philosophie dar, und diese Philosophie ist überholt. « ${ }^{15}$

Der Rationalismus hat nur dann einen Wert, wenn er sich »spezialisiert ", wie Bachelard in einer berühmten Rede vor der »Société française de philosophie « feststellte: »Doch muss man anmerken, dass die Spezialisierung - das wollte ich sagen - eine Notwendigkeit ist, und zwar eine glückliche Notwendigkeit! Erst durch Spezialisierung kommt der rationalistische Muskeltonus zustande. ${ }^{16}$ Ebenso hebt er in L'activité rationaliste de la physique contemporaine hervor: »Eine Wissenschaftskultur ohne Spezialisierung wäre ein wirkungsloses Werkzeug, eine Schere mit stumpfen Schneiden. ${ }^{17}$ Die Geschichte der Wissenschaften wird auf diese Weise zur Geschichte der Spezialisierungen des Wissens: Es gibt nicht nur eine einzige wissenschaftliche Methode, sondern so viele Methoden, wie es unterschiedliche Wissenschaften gibt. Diese durch die Wissenschaften eröffneten "rationalen Regionen «stehen in einer gewissen Analogie zum husserlschen Begriff der »regionalen Ontologien «. Der Irrtum Husserls bestand nach Bachelard jedoch darin, dass er diese regionalen Ontologien unter einer einzigen eidetischen Wissenschaft der physischen Natur im Allgemeinen vereinte.

Die Vernunft hat der Wissenschaft zu gehorchen und ist daher ohne Unterlass in Bewegung. Häufig wird die Formulierung aus L'ean et les rêves angeführt: »Vernünftig? - Wir bemühen uns, es zu werden. «18 Dies impliziert ohne Zweifel eine Bewegung in Richtung einer Universalisierung: »In jedem regionalen Rationalismus gibt es einen Kern eines allgemeinem Rationalismus, ohne den ich auch nicht von Rationalismus spräche. «19 Der Begriff des »regionalen Rationalismus « kam, wie aus dem Angeführten deutlich wird, im Kontext einer allgemeinen Debatte auf, die in diesen Jahren um die Frage des Rationalismus geführt wurde. Bachelards Vortrag vor der »Société française de philosophie « von 1950 ist in gewissem Sinn eine Antwort auf einen Vortrag von Julien Benda, den dieser 1947 vor derselben Gesellschaft hielt. Unter dem Titel »Über einige wissenschaftlichen Konstanten des menschlichen Geistes " nahm 
Benda es mit sämtlichen Versuchen einer » dynamischen`Geschichte der Vernunft " auf, wobei er zunächst Léon Brunschvicg und dessen Buch Les âges de l'intelligence im Auge hatte, das »eine Art von Charta dieser Philosophie « darstelle. ${ }^{20}$ Seine Kritik schließt aber auch die Vertreter einer »mobilistischen « Philosophie mit ein, zu denen Bachelard, aber auch Abel Rey, Edouard Le Roy, Louis Rougier oder Louis Lavelle zählten, die allesamt, zum großen Erstaunen mancher, als Erben von Bergsons "Mobilismus « identifiziert werden: Diese Philosophen seien zwar » recht unterschiedlich in ihrer Gesamthaltung ${ }^{21}$ doch träfen sie sich in der Vorstellung, dass das Denken Bewegung sei, sowie in ihrem »Willen zur Ablehnung aller Beständigkeit des wissenschaftlichen Geistes «, indem sie vorgäben, »dass dieser Geist je nach Ort variiert «. ${ }^{22}$ In einem in Anschluss an Bachelards Vortrag veröffentlichten Brief kam Benda auf seinen Anklagepunkt zurück und schrieb, »der Suprarationalismus des Herrn Bachelard scheint mir genauso wie der Transrationalismus des Herrn Ruyer in keinster Weise eine Sublimierung des Rationalismus darzustellen, sondern eher dessen Negation «. Und ferner: »Die Vernunft ist unnachgiebig (was nicht heißen will, sie sei ohne Nuancen), oder sie ist nichts. $\ll^{23}$

\section{CANGUILHEM UND DER VITALE RATIONALISMUS}

Auch bei Canguilhem findet sich, unabhängig von Bachelard formuliert, die Idee eines regionalen Rationalismus. So erklärt er in seiner Schrift "Zur Lage der biologischen Philosophie in Frankreich « aus dem Jahre 1947, dass man »eine Geschichte und Geographie der Rationalisten « betreiben und begreifen müsste, dass es unterschiedliche Formen gibt, Vernunft anzuwenden, und verweist als Beispiel auf Frankreich und Deutschland. ${ }^{24}$ Canguilhem kritisiert den auf mathematischer Grundlage errichteten »traditionellen französischen Rationalismus «, wie er etwa von Brunschvicg vertreten wurde, zumal dessen Philosophie niemals für die Originalität des Lebendigen empfänglich gewesen sei: »Wegen Descartes oder, genauer gesagt, seit Descartes gilt die biologische Philosophie bei uns als äußerst suspekte Spekulation. Dem Leben wird im kartesianischen System keine ontologische Qualität beigemessen. ${ }^{25}$ Umgekehrt könnte es sein, »daß die Deutschen ihren Rationalismus anders anwenden als wir «, da sie, angetrieben von einem »Phänomen der kollektiven biologischen Energie «, möglicherweise empfänglicher seien für das Thema des Lebens. ${ }^{26}$ Canguilhem zufolge kann man 
sehr wohl Rationalist sein, ohne zugleich die Originalität des Lebens zu leugnen: "Das ist übrigens so wahr, daß der Rationalismus nicht rein bleiben kann, wenn er fruchtbar sein will. « ${ }^{27}$ Es gibt demnach einen vitalen Rationalismus, der von der Biologie inspiriert ist.

Die Bedeutung, den der Begriff des regionalen Rationalismus bei Bachelard einnimmt, schätzt Canguilhem unterschiedlich ein, das heißt, er sieht in diesem nicht immer eines der zentralen Konzepte im Werk Bachelards: Während er in zwei Artikeln über Bachelard den Begriff des epistemologischen Regionalismus gar nicht erwähnt, erklärt er ihn in zwei anderen zum Herz des bachelardschen Denkens. So stellt er beispielsweise in einem für eine italienische Enzyklopädie verfassten Artikel über Bachelard, als eine dessen zentralen Thesen heraus, dass es »eine philosophische Kritik nur seitens einer angewandten Vernunft gibt und dass jede Anwendung notwendigerweise regional ist «. ${ }^{28}$ Desgleichen merkt er an anderer Stelle an: »Der Nicht-Kartesianismus und der Nicht-Kantianismus dieser Epistemologie werden noch manifester in der Anerkennung einer Vielfalt von Rationalismen, in der Bildung regionaler Rationalismen, d. h. in der Bestimmung der Grundlagen partikularer Wissensbereiche. ${ }^{29}$

Canguilhem tendiert sogar dazu, diese Auffassung zu verallgemeinern, denn er spricht nicht nur von »regionalen Epistemologien«, sondern auch von »regionalen Wissenschaftsgeschichten«. Wie er in einer von ihm zusammengestellten Sammlung von Texten zur Geschichte der Wissenschaften von 1970 erklärt, muss die Wissenschaftsgeschichte selbst regionalisiert werden: »Aufgegeben ist der Traum von einer allgemeinen Wissenschaftsgeschichte; es gibt keine Geschichte der Wissenschaften, sondern Geschichten der Wissenschaften. Wir stehen also regionalen Geschichten gegenüber, von denen jede einzelne auf eine unterschiedliche Auffassung dessen rekurriert, was eine Geschichte der Wissenschaften zu sein hat. ${ }^{30}$ Es gibt also genauso viele Arten, die Geschichte der Wissenschaften zu schreiben, wie es Wissenschaften gibt. Die Methoden der Wissenschaftsgeschichtsschreibung sind für die Biologie und die mathematische Physik nicht dieselben, und Canguilhem führt auf diesen Unterschied der Gegenstände auch die Differenzen zurück, die zwischen seiner eigenen Epistemologie (der Biologie) und Bachelards Epistemologie (der Physik) bestehen. Canguilhem zufolge ist die Epistemologie »im Gegensatz zur Wissenschaftsphilosophie eine spezielle oder auf bestimmte Bereiche begrenzte [régionale] Untersuchung « ${ }^{31}$ Von diesem Standpunkt aus kritisiert er explizit den Redukti- 
onismus des Wiener Kreises: Keine Wissenschaft könne per Klassifikation auf eine vorausgehende Wissenschaft reduziert werden. Desgleichen lehnt er das "positivistische « Bild einer »Pyramide der Wissenschaften « ab. Die nicht-positivistische Epistemologie, d. i. seine eigene, »hat die Vorstellung von der Stufenleiter durch diejenige von der Fläche ersetzt. Der Zusammenhang zwischen den Wissenschaften ist damit zu einer netzförmigen Verknüpfung geworden. « ${ }^{32}$

Ein letzter wichtiger Aspekt des »epistemologischen Regionalismus « bei Canguilhem ist dessen Gebrauch räumlicher Metaphern im Kontext des Begriffs der wissenschaftlichen Ideologie, etwa der Vorstellungen vom »Erkenntnisraum «, von »Grenzen «, von »Verlagerung « und vom »Import « von Begriffen. In seinem Artikel über wissenschaftliche Ideologien betont Canguilhem den Begriff des »Ortes", der von einer Wissenschaft eingenommen wird: »Wenn eine Wissenschaft sich anschickt, einen Ort einzunehmen, den ihr die Ideologie zugewiesen zu haben scheint, geschieht dies nicht dort, wo man es erwarten würde.«33 Diese Verlagerungen finden jedoch nicht nur im Raum, sondern auch in der Zeit statt, wie von anderen Metaphern angezeigt wird, die auf »Filiationen « anspielen, auf historische Verlagerungen verschiedener »Achsen $«$.

Die Debatte rund um den epistemologischen Rationalismus nahm also ihren Ausgang im Umfeld des Rationalismusproblems. Es stellt sich nun die Frage, ob eine solche Sichtweise das traditionelle Verständnis des Rationalismus nur modifizierte, oder ob sie dieses nicht vielmehr völlig zerstörte. Bachelard wollte offensichtlich den Rationalismus retten und auch Canguilhem hielt weiterhin an diesem fest, wenngleich er die Vernunft neu definierte: »Wenn man unter Vernunft weniger ein Vermögen zur Wahrnehmung der wesentlichen Beziehungen versteht, die in der Realität der Dinge oder des Geistes enthalten sind, denn vielmehr als ein Vermögen zur Herstellung von normgebenden Beziehungen in der Erfahrung des Lebens, dann wollen auch wir uns in diesem Sinne als vernünftig [rationaliste] bezeichnen. ${ }^{34}$

\section{ALTHUSSER UND FOUCAULT: KONTINENTE UND TERRITORIEN}

Zwei Leser Bachelards und Canguilhems, nämlich Louis Althusser und Michel Foucault, haben den Begriff des regionalen Rationalismus wieder aufgegriffen und transformiert. Es ließe sich noch ein weiterer Leser anführen, und zwar Pierre Bourdieu, der sich aber darauf beschränkte, 
ihn zu popularisieren, insbesondere in seiner berühmten Einführung in die Soziologie, Soziologie als Beruf (1968). Sehr getreu trägt Bourdieu hier Bachelards Standpunkt vor und übernimmt ihn für die Soziologie: Die Epistemologie Bachelards »verwirft den Formalismus und das Beharren auf Konstanz der einen und unteilbaren Vernunft zugunsten eines Pluralismus von Rationalismen, die den jeweils von ihnen rationalisierten Wissenschaftsbereichen entsprechen", und eignet sich daher »auch hervorragend dafür, den Sozialwissenschaften, die besonders gefährliche Erkenntnishindernisse zu überwinden haben, um ihren eigenen, regionalen Rationalismus begründen zu können, eine Sprache zu geben und theoretischen Beistand zu leisten $« .{ }^{35}$

Althusser wiederum übernahm die explizit von Bachelard entliehene geographische Metapher, transformierte sie aber zugleich, indem er in seinem berühmten Vortrag über »Lenin und die Philosophie (erneut vor der »Société française de philosophie«) anstatt von »Regionen « zu sprechen, auf »Kontinente « abhob: » Wenn wir die großen wissenschaftlichen Entdeckungen der menschlichen Geschichte betrachten, so scheint es, als könnten wir das, was wir als >Wissenschaften $<$ bezeichnen, als regionale Formationen dem zuordnen, was wir die großen theoretischen Kontinente nennen wollen. « ${ }^{36}$ Vor Marxens Entdeckung hätten schlichtweg nur zwei Kontinente existiert - »der Kontinent Mathematik «, der durch die Griechen erschlossen wurde, und »der Kontinent Physik «, der durch Galilei und seine Nachfolger erschlossen wurde. Disziplinen wie die Chemie oder Biologie wären einfach als Bereiche dieses zweiten großen Kontinents der Physik verstanden worden. Erst Marx hätte den »Kontinent Geschichte« erschlossen und Freud stünde zweifelsohne am Beginn der Entdeckung eines »neuen Kontinent[s], mit dessen Erforschung wir gerade erst beginnen «. ${ }^{37}$

Indem er die Metapher des Kontinents verwendet, möchte Althusser besonders den andersartigen Charakter dieser Räume hervorheben. Denn im Unterschied zu Regionen sind Kontinente völlig unverbunden. In Beantwortung einer Frage Paul Ricœurs beruft sich Althusser gegen Engels diesmal auf Spinoza: »Es ist Engels, der von der Verkettung der Regionen gesprochen hat [...], ich aber überhaupt nicht. Es gibt Kontinente und ich behaupte überhaupt nicht, dass sie gemeinsame Grenzen hätten: Ich bin Spinozist; es gibt eine Unendlichkeit von Attributen ... . « ${ }^{38}$

Zugleich war sich Althusser der Schwierigkeiten bewusst, die die Verwendung solcher Metaphern zur Behandlung vorgeblich wissenschaftlicher Fragen mit sich bringt. So merkte er mit Blick auf Foucault 
an: »Die Verwendung solcher Raummetaphern wie Feld, Terrain, Raum, Situierung, Position etc. stellt ein theoretisches Problem dar: das Problem ihrer Berechtigung in einem Diskurs mit wissenschaftlichem Anspruch. Das Problem kann folgendermaßen formuliert werden: Warum ist die Verwendung von Metaphern nichtwissenschaftlicher Provenienz in einer bestimmten Form wissenschaftlicher Rede unumgänglich? « ${ }^{39}$ Was seine eigene Metapher von den Kontinenten anbelangt, äußert sich Althusser weniger scharf und überlässt es ironischerweise seinen Lesern, ihre Schlüsse daraus zu ziehen: »Ich überlasse es Ihnen, all diese Metaphern in einer provisorischen, aber notwendigen Arbeit zusammenzuflicken. ${ }^{40}$

Insbesondere im Hinblick auf Foucaults Arbeiten stellte sich Althusser die Frage nach der wissenschaftlichen Konsistenz geographischer Metaphern. Natürlich war Foucault von Bachelard inspiriert. So erklärte er ausdrücklich: »[I]n seinen Überlegungen zur Diskontinuität in der Geschichte der Wissenschaften und im Gedanken, dass die Vernunft, indem sie die Gegenstände ihrer Analyse selbst konstituiert, an sich selbst arbeitet, gibt es eine ganze Reihe von Elementen, von denen ich profitieren konnte und die ich aufgenommen habe. ${ }^{41}$ Wenn es die Archäologie erlaubt, die zugrunde liegenden »Begriffsschichten « in dieser oder jener Episteme wiederzufinden, dann erlaubt es der Begriff der »Region« kraft seiner »ent-einheitlichenden « und zerstreuenden Wirkung, jene Deutung zu vermeiden, die eine »einheitliche « Auffassung der Archäologie hervorbringen würde, nämlich eine vereinheitlichende Tafel von Begründungen. In der Einleitung zur englischen Übersetzung der Ordnung der Dinge gibt Foucault zu bedenken, dass seine Untersuchung »keine Analyse [...] einer bestimmten Weltanschauung ", sondern »eine streng >regionale< Untersuchung ist $"{ }^{42}$ In der Archäologie des Wissens betont er ebenfalls: Der archäologische »Vergleich ist [...] immer begrenzt und regional. Weit davon entfernt, allgemeine Formen erscheinen zu lassen zu wollen, versucht die Archäologie einzelne Konfigurationen aufzuzeichnen. « ${ }^{43}$

Was aber die besondere Originalität Foucaults ausmacht, ist der politische Charakter, den er seinen Verweisen auf Wissensregionen verleiht und den er vermittels des Begriffs des »Territoriums « zum Ausdruck bringt. In einem Gespräch mit Geographen gesteht er ein, von Raummetaphern »besessen « gewesen zu sein, doch, so erklärt er, sei dies dem Umstand ihrer politischen Anklänge geschuldet gewesen: »So wie man das Wissen als Region, Gebiet, Einpflanzung, Verlegung oder 
Übertragung analysieren kann, kann man den Prozess erfassen, durch den das Wissen als eine Macht funktioniert und deren Wirkungen verstärkt. « ${ }^{44}$ Räumliche Metaphern seien politisch, während zeitliche Metaphern auf die Idee eines individuellen Bewusstseins verweisen, das seine eigene Zeitlichkeit hätte, und somit auch eine Einheit des Bewusstseins voraussetzen würde.

Zusammenfassend kann festgehalten werden, dass die Debatte über den epistemologischen Rationalismus im Falle von Bachelard und Canguilhem auf eine allgemeinere Kontroverse um den Rationalismus und im Falle von Althusser und Foucault auf im weiteren Sinne politische Überlegungen verweist. Analoge Debatten über räumliche Repräsentationen des Wissens fanden auch im Kontext des Wiener und später angelsächsischen Neopositivismus statt; sie nahmen jedoch andere Formen an. Man wird sich an die Kritik erinnern, die Otto Neurath an der "pyramidenförmigen « Repräsentation des »Systems « des Wissens formulierte, die er Rudolf Carnap zuschrieb und der er selbst eine »enzyklopädische « Sichtweise gegenüberstellte: Für ihn sei es »absurd von einem einzigen und totalen System der Wissenschaften zu sprechen«; vielmehr müsse man sich »innerhalb eines stets veränderlichen Rahmens, jenes einer Enzyklopädie « bewegen. ${ }^{45}$ Neurath verfolgte zugleich politische Absichten, und so stand der Kampf um die "Einheit der Wissenschaften « allein im Dienste politischer Zielsetzungen, so dass die angestrebte Vereinigung als » Weg zum Sozialismus « erscheinen konnte. ${ }^{46}$

In den vergangenen Jahren fand im angelsächsischen Raum eine Debatte rund um die Vorstellung der »disunity of sciences « und der »Vielfalt der Stile wissenschaftlichen Denkens « statt, die in Frankreich kaum Widerhall fand. ${ }^{47}$ Wenn diese Auseinandersetzung in Frankreich kaum zur Entfaltung gekommen ist, lässt sich hierfür die Hypothese aufstellen, dass die hiesige Wissenschaftsphilosophie weitestgehend auf der Philosophie von Auguste Comte gründet, für den die Verschiedenartigkeit wissenschaftlicher "Methoden der Untersuchung " selbstverständlich ist. Jede Wissenschaft verfüge über ihre eigene Untersuchungsmethode in Abhängigkeit ihres Anwendungsbereiches: "Das Studium der Methode lässt sich nicht von dem der Untersuchungen trennen, wo sie zur Anwendung gekommen ist. "Sie müssen »in actu « untersucht werden. ${ }^{48}$ In ihren Details erinnern die comteschen »Methoden der Untersuchung " sehr deutlich an Alistair Crombies oder Ian Hackings »Stile wissenschaftlichen Denkens «. Diese »regionalistische« und anti- 
kartesische Dimension der Philosophie Comtes ist bislang sicherlich unterschätzt worden. Gleichwohl stellt sie die Grundlage überhaupt der Geschichte der französischen Epistemologie dar.

Aus dem Französischen von Konrad Petrovszky

\section{A N M ER K U N G E N}

1 Nach meinem Dafürhalten findet sich diese Wendung zum ersten Mal 1907 in Abel Reys Buch La théorie de la physique chez les physiciens contemporains (Paris: Félix Alcan, 1907), dt. Die Theorie der Physik bei den modernen Physikern (Leipzig: Klinkhardt, 1908). 1932 gründete Abel Rey das »Institut d'histoire des sciences et des techniques ", das nach ihm von Bachelard und später von Canguilhem geleitet wurde.

2 François Dagognet, "A Regional Epistemology with Possibilities for Expansion «, Science in Context, 9.1 (1996), S. 5-14.

3 Francis Bacon, Über die Würde und die Förderung der Wissenschaften. London 1605/1623, übers. v. Jutta Schlösser (Freiburg: Haufe, 2006), S. 555-56.

4 Francis Bacon, Neu-Atlantis, hg. v. Beate Behrens (Berlin-Ost: Akademie-Verlag, 1984), S. 40-41.

5 Bacon, Über die Würde, S. 127.

6 Jean le Rond d'Alembert, Einleitung zur Enzyklopädie, hg. v. Günther Mensching (Hamburg: Meiner, 1997), S. 42.

7 Gottfried Wilhelm Leibniz, Schöpferische Vernunft. Schriften aus den Jahren 1668-1686, übers. u. hg. v. Wolf von Engelhardt (Marburg: Simons, 1951), S. 204

8 Georges Canguilhem, »Essais. Esquisse d'une politique de paix. Préambule«, Libres propos (Journal d'Alain), 20 (März 1929), jetzt wiederabgedruckt in ders., Euvres complètes I: Écrits philosophiques et politiques (1926-1939) (Paris: Vrin, 2011), S. 213-16.

9 Paul Vidal de la Blache, »Régions françaises «, Revue de Paris, 6 (1910), S. 821-49.

10 Georges Canguilhem, "Cours de 1939-1940 à l'Institut agronomique de Toulouse «, zitiert nach J.-F. Braunstein, »A la découverte d'un `Canguilhem perdu«", in Canguilhem, Euvres complètes I, S. 125.

11 Neuere Kritiken an der historischen Epistemologie sparen nicht mit diesbezüglichen Anspielungen. Vgl. etwa Pierre Jacob, »Il regionalismo epistemologico: una tendenza delle filosofia contemporanea delle scienze in Francia ", Rivista di filosofia, 82 (1991), S. 279-300.

12 Gaston Bachelard, Der neue wissenschaftliche Geist, übers. v. Michael Bischoff (Frankfurt a.M.: Suhrkamp, 1988), S. 174.

13 Gaston Bachelard, "Le surrationalisme", in ders., L'engagement rationaliste (Paris: PUF, 1972), S. 7-12, hier S. 7.

14 Ebd., S. 8. In Analogie dazu hatte sich bereits André Breton von Bachelard inspiriert, um in Bezug auf ihn von einem "Suprarationalismus « zu sprechen, der den 
Surrealismus »verdoppele" und »vermesse (André Breton, "Crise de l'objet ", Cahiers d'art, 1-2 [1936], S. 21-25).

15 Gaston Bachelard, Die Philosophie des Nein. Versuch einer Philosophie des neuen wissenschaftlichen Geistes, übers. v. Gerhard Schmidt u. Manfred Tietz (Wiesbaden: Heymann, 1978), S. 165.

16 Gaston Bachelard, »De la nature du rationalisme«, in ders., L'Engagement rationaliste, S. 45-88, hier S. 55.

17 Gaston Bachelard, L'activité rationaliste de la physique contemporaine (Paris: PUF 1951), S. 12.

18 Gaston Bachelard, L'ean et les rêves. Essai sur l'imagination de la matière (1942) (Paris: Biblio Essais, 2005), S. 14. Dies ist auch das erste Bachelard-Zitat Canguilhems, das dieser in seinem Aufsatz "Note sur la situation faite en France à la philosophie biologique von 1947 anführt. Die deutsche Übersetzung findet sich unter dem Titel »Zur Lage der biologischen Philosophie in Frankreich« in Georges Canguilhem, Wissenschaft, Technik, Leben. Beiträge zur historischen Epistemologie, übers. v. Ronald Vouillé u. a., hg. u. mit einem Nachwort v. Henning Schmidgen (Berlin: Merve, 2006), S. 23-39.

19 Bachelard, »De la nature «, S. 69.

20 Julien Benda, "De quelques constantes de l'esprit scientifique à travers l'histoire", Bulletin de la Société Française de Philosophie, 42 (1948), S. 93-126, hier S. 95.

21 Ebd., S. 94.

22 Ebd., S. 113.

23 »Lettre de Julien Benda «, im Anhang zu Bachelard, »De la nature«, S. 86.

24 Canguilhem, »Zur Lage«, S. 33.

25 Ebd., S. 26.

26 Ebd., S. 28 u. 29.

27 Ebd., S. 31.

28 Georges Canguilhem, "Gaston Bachelard «, in Scienziati e tecnologi contemporanei, hg. v. Gino Cesaretti, 3 Bde. (Milano: Mondadori, 1974-75), I (r974), S. 65-67, hier S. 66.

29 Georges Canguilhem, »Dialectique et philosophie du non chez Gaston Bachelard ", in ders., Études d'histoire et de philosophie des sciences, 7. erw. Aufl. (Paris: Vrin, 1994), S. 196-207, hier S. 201.

30 Georges Canguilhem, »La méthode en histoire des sciences ", in Introduction à l'histoire des sciences, hg. v. Suzanne Bachelard u. a., 2 Bde. (Paris: Hachette, 1970), II: Objet, méthode, exemple, S. 30-32, hier S. 31.

31 Georges Canguilhem, »Philosophie und Wissenschaft: Gespräch mit Alain Badiou (1964) «, in ders., Wissenschaft, Technik, Leben, S. 49-68, hier S. 49.

32 Georges Canguilhem, »Der epistemologische Status der Medizin", in ders., Grenzen medizinischer Rationalität. Historisch-epistemologische Untersuchungen, übers. v. Monika Noll, hg. v. Gerd Herrmann (Tübingen: edition diskord, 1989), S. 69-93, hier S. 86.

33 Georges Canguilhem, »Qu'est-ce qu'une idéologie scientifique? «, in ders., Idéo- 
logie et rationalité dans l'histoire des sciences de la vie (Paris: Vrin, 1977), S. 33-45, hier S. 40.

34 Ders., "Zur Lage«, S. 39.

35 Pierre Bourdieu, Jean-Claude Chamboredon u. Jean-Claude Passeron, Soziologie als Beruf. Wissenschaftstheoretische Voraussetzungen soziologischer Erkenntnis, übers. v. Hella Beister u. a. (Berlin, New York: de Gruyter, 1991), S. 93.

36 Louis Althusser, "Lenin und die Philosophie", in ders., Lenin und die Philosophie, übers. v. Klaus-Dieter Thieme (Reinbek bei Hamburg: Rowohlt, 1974), S. 7-46, hier S. 19.

37 Ebd., S. 19-20.

38 Louis Althusser, "Lénine et la philosophie ", Bulletin de la Société française de philosophie, 62.4 (1968), S. 127-83, hier S. 164 (die später abgedruckte und auch ins Deutsche übersetzte veränderte Fassung des Vortrags enthält den Diskussionsteil nicht [Anm. d. Übers.]).

39 Louis Althusser, "Einführung. Vom 'Kapital zur Philosophie von Marx«, in Das Kapital lesen, hg. v. Louis Althusser u. Étienne Balibar, 2 Bde. (Reinbek bei Hamburg: Rowohlt, 1972), I, S. 11-93, hier S. 30, Anm. 7.

40 Louis Althusser, »Über die Beziehung von Hegel zu Marx«, in ders., Lenin und die Philosophie, S. 47-67, hier S. 49.

41 Michel Foucault, »Gespräch mit Ducio Trombadori«, in ders., Schriften in vier Bänden. Dits et Ecrits, hg. v. Daniel Defert u. François Ewald (Frankfurt a.M.: Suhrkamp, 2001-05), Iv: 1980-1988, übers. v. Michael Bischoff u. a. (2002), S. 51-119, hier S. 70.

42 Michel Foucault, "Vorwort zur englischen Ausgabe", in ders., Schriften in vier Bänden, II: 1970-1975 (2002), S. 9-16, hier S. 10.

43 Michel Foucault, Archäologie des Wissens, übers. v. Ulrich Köppen (Frankfurt a.M.: Suhrkamp, 1981), S. 225.

44 Michel Foucault, »Fragen an Michel Foucault zur Geographie«, in ders., Schriften in vier Bänden, III: 1976-1979 (2003), S. 38-54, hier S. 45.

45 Otto Neurath, "Die Enzyklopädie als `Modell«", in Wiener Kreis. Texte zur wissenschaftlichen Weltauffassung von Rudolf Carnap, Otto Neurath, Moritz Schlick, Philipp Frank, Hans Hahn, Karl Menger, Edgar Zilsel und Gustav Bergmann, hg. v. Michael Stöltzner u. Thomas Uebel (Hamburg: Meiner, 2006), S. 375-95, hier S. 376.

46 Jordi Cat, Hasok Chang u. Nancy Cartwright, »Otto Neurath. Unification as the Way to Socialism «, in Einheit der Wissenschaften, hg. v. Jürgen Mittelstraß (Berlin: de Gruyter, 1991), S. 91-110.

47 The Disunity of Science. Boundaries, Contexts, and Power, hg. v. Peter Galison u. David J. Stump (Stanford: Stanford University Press, 1996).

48 Die positive Philosophie von Auguste Comte im Auszug von Jules Rig, übers. v. J. H. v. Kirchmann, 2 Bde. (Leipzig: Dürr, 1883), I, S. 14. [Diese bereits ältere, aber für spätere Auflagen herangezogene Übersetzung verschleift des Öfteren die Sätze miteinander; so unterschlägt sie etwa die hier von Braunstein hervorgehobene Passage Comtes »c'est en action qu'il faut la considérer « in Auguste Comte, Cours de philosophie positive (Paris: Hermann, 1998), I, 1ère leçon, S. 38; Anm. 
d. Übers.]. Siehe dazu Jean-François Braunstein, »La philosophie des sciences d'Auguste Comte «, in Les philosophes et la science, hg. v. Pierre Wagner (Paris: Gallimard, 2002), S. 787-822. 
Jean-François Braunstein, »Die Geschichte des Regionsbegriffs in der Epistemologie $\ll$, in Situiertes Wissen und regionale Epistemologie: Zur Aktualität Georges Canguilhems und Donna J. Haraways, hg. v. Astrid Deuber-Mankowsky und Christoph F. E. Holzhey, Cultural Inquiry, 7 (Wien: Turia + Kant, 2013), S. 35-49 <https: //doi.org/10.37050/ci-07_02>

\section{REFERENCES}

Althusser, Louis, Lenin und die Philosophie, übers. v. Klaus-Dieter Thieme (Reinbek bei Hamburg: Rowohlt, 1974)

$\gg$ Einführung. Vom >Kapital< zur Philosophie von Marx «, in Das Kapital lesen, hg. v. Louis Althusser u. Étienne Balibar, 2 Bde. (Reinbek bei Hamburg: Rowohlt, 1972), I, S. 11-93 »Lénine et la philosophie «, Bulletin de la Société française de philosophie, 62.4 (1968), S. 127 83

Bachelard, Gaston, L'activité rationaliste de la physique contemporaine (Paris: PUF, 1951)

_L'eau et les rêves. Essai sur l'imagination de la matière (1942) (Paris: Biblio Essais, 2005)

L'engagement rationaliste (Paris: PUF, 1972)

Der neue wissenschaftliche Geist, übers. v. Michael Bischoff (Frankfurt a.M.: Suhrkamp, 1988)

- Die Philosophie des Nein. Versuch einer Philosophie des neuen wissenschaftlichen Geistes, übers. v. Gerhard Schmidt u. Manfred Tietz (Wiesbaden: Heymann, 1978)

Bacon, Francis, Neu-Atlantis, hg. v. Beate Behrens (Berlin-Ost: Akademie-Verlag, 1984)

— Über die Würde und die Förderung der Wissenschaften. London 1605/1623, übers. v. Jutta Schlösser (Freiburg: Haufe, 2006)

Benda, Julien, $\gg$ De quelques constantes de l'esprit scientifique à travers l'histoire $\ll$, Bulletin de la Société Française de Philosophie, 42 (1948), S. 93-126

Bourdieu, Pierre, Jean-Claude Chamboredon u. Jean-Claude Passeron, Soziologie als Beruf. Wissenschaftstheoretische Voraussetzungen soziologischer Erkenntnis, übers. v. Hella Beister u. a. (Berlin, New York: de Gruyter, 1991) <https://doi.org/10.1515/ 9783110856477>

Braunstein, Jean-François, $\gg$ La philosophie des sciences d'Auguste Comte $\ll$, in Les philosophes et la science, hg. v. Pierre Wagner (Paris: Gallimard, 2002), S. 787-822

Breton, André, »Crise de l'objet «, Cahiers d'art, 1-2 (1936), S. 21-25

Canguilhem, Georges, Grenzen medizinischer Rationalität. Historisch-epistemologische Untersuchungen, übers. v. Monika Noll, hg. v. Gerd Hermann (Tübingen: edition diskord, 1989)

- Idéologie et rationalité dans l'histoire des sciences de la vie (Paris: Vrin, 1977)

Wissenschaft, Technik, Leben. Beiträge zur historischen Epistemologie, übers. v. Ronald Vouillé u. a., hg. u. mit einem Nachwort v. Henning Schmidgen (Berlin: Merve, 2006)

— $\gg$ Dialectique et philosophie du non chez Gaston Bachelard «, in ders., Études d'histoire et de philosophie des sciences, 7. erw. Aufl. (Paris: Vrin, 1994), S. 196-207

— $\gg$ Gaston Bachelard «, in Scienziati e tecnologi contemporanei, hg. v. Gino Cesaretti, 3 Bde. (Milano: Mondadori, 1974-75), I (1974), S. 65-67

— $\gg$ La méthode en histoire des sciences «, in Introduction à l'histoire des sciences, hg. v. Suzanne Bachelard u. a., 2 Bde. (Paris: Hachette, 1970), II: Objet, méthode, exemple, S. 30-32

Euvres complètes, hg. v. Jacques Bouveresse, Jean-François Braunstein, Claude Debru, Anne Fagot-Largeault, Camille Limoges u. Yves Schwartz (Paris: Vrin, 2011-), I: Écrits philo- 
sophiques et politiques (1926-1939), hg. v. Jean-François Braunstein u. Yves Schwartz unter Mitw. v. Michele Cammelli u. Xavier Roth, Vorwort v. Jacques Bouveresse (2011)

Cat, Jordi, Hasok Chang u. Nancy Cartwright, »Otto Neurath. Unification as the Way to Socialism «, in Einheit der Wissenschaften, hg. v. Jürgen Mittelstraß (Berlin: de Gruyter 1991), S. $91-110$

Comte, Auguste, Cours de philosophie positive (Paris: Hermann, 1998)

—Die positive Philosophie von Auguste Comte im Auszug von Jules Rig, übers. v. J. H. v. Kirchmann, 2 Bde. (Leipzig: Dürr, 1883)

De la Blache, Paul Vidal, »Régions françaises «, Revue de Paris, 6 (1910), S. 821-49

Dragonet, François, »A Regional Epistemology with Possibilities for Expansion «, Science in Context, 9.1 (1996), S. 5-14 <https://doi.org/10.1017/S0269889700002271>

D’Alembert, Jean le Rond, Einleitung zur Enzyklopädie, hg. v. Günther Mensching (Hamburg: Meiner, 1997)

Foucault, Michel, Archäologie des Wissens, übers. v. Ulrich Köppen (Frankfurt a.M.: Suhrkamp, 1981)

_ Schriften in vier Bänden. Dits et Ecrits, hg. v. Daniel Defert u. François Ewald (Frankfurt a.M.: Suhrkamp, 2001-05)

Galison, Peter u. David J. Stump (Hg.), The Disunity of Science. Boundaries, Contexts, and Power (Stanford: Stanford University Press, 1996)

Jacob, Pierre, $\gg$ Il regionalismo epistemologico: una tendenza delle filosofia contemporanea delle scienze in Francia «, Rivista di filosofia, 82 (1991), S. 279-300

Leibniz, Gottfried Wilhelm, Schöpferische Vernunft. Schriften aus den Jahren 1668-1686, übers. u. hg. v. Wolf von Engelhardt (Marburg: Simons, 1951)

Neurath, Otto, $\gg$ Die Enzyklopädie als $>$ Modell $<\ll$, in Wiener Kreis. Texte zur wissenschaftlichen Weltauffassung von Rudolf Carnap, Otto Neurath, Moritz Schlick, Philipp Frank, Hans Hahn, Karl Menger, Edgar Zilsel und Gustav Bergmann, hg. v. Michael Stöltzner u. Thomas Uebel (Hamburg: Meiner, 2006), S. 375-95

Reys, Abel, La théorie de la physique chez les physiciens contemporains (Paris: Félix Alcan, 1907), dt. Die Theorie der Physik bei den modernen Physikern (Leipzig: Klinkhardt, 1908) 